\title{
A Combined Computational and Experimental Study on Nanoparticle Transport and Partitioning in the Human Trachea and Upper Bronchial Airways
}

\author{
Jiawei Ma1, Wei-Chung Su ${ }^{2 * *}$, Yi Chen², Yidan Shang ${ }^{1}$, Jingliang Dong ${ }^{1}$, Jiyuan Tu ${ }^{1}$, Lin Tian ${ }^{*}$ \\ ${ }^{1}$ School of Engineering - Mechanical and Automotive, RMIT University, Bundoora, VIC, Australia \\ ${ }^{2}$ School of Public Health, Department of Epidemiology, Human Genetics, and Environmental Sciences, The University of \\ Texas - Health Science Center at Houston, TX, USA
}

\begin{abstract}
In the past few decades, the transport and deposition of aerosol in the human respiratory tract has been a crucial area of research, resulting in the identification of the toxicity pathways of inhaled pollutants and facilitating the design of efficient drug delivery systems for targeted treatment. Owing to the complexity of the tracheobronchial tree, experimental studies in vivo/in vitro have been extremely limited; hence, detailed data on the airflow and particle dynamics have been obtained predominantly through computational investigations. With rapid advances in medical imaging and computational capacities, sophisticated human tracheobronchial trees that include the $6^{\text {th }}, 7^{\text {th }}$ or $15^{\text {th }}$ generation have been increasingly described in the literature. However, continued progress in anatomical reconstruction and mathematical idealized modeling, the two most frequently employed approaches to airway modeling, requires a detailed fundamental analysis on the morphology-induced sensitivity of particle-flow partitioning, and particle deposition in the airways. This study combined numerical and experimental investigations on the transport, deposition and partitioning of nanoparticles in the upper tracheobronchial airways. An anatomically realistic airway was reconstructed via CT scans, and a simplified numerical model was developed that incorporated physical irregularities in the trachea and assessed new boundary conditions to simulate air partitioning in the lobar bronchi, and flow and particle dynamics. An experiment measuring the penetration and deposition of sodium chloride $(\mathrm{NaCl})$ nanoparticles in the anatomical and idealized airway models was conducted in parallel, and the results were compared with the computational predictions.
\end{abstract}

Keywords: Airway morphology; Nanoparticle transport; Deposition and partitioning; Tracheobronchial tree; Computational modeling; Experimental measurement.

\section{INTRODUCTION}

Aerosol transport and deposition in human respiratory airways has been an important research area in past few decades. The knowledge of particle trajectory, particle-flow interaction and deposition site is of significant value to respiratory toxicity study, and also to the therapeutic design of efficient inhalable drug delivery devices (Kleinstreuer and Zhang, 2003; Asgharian and Price, 2007; Inthavong et al., 2010; Longest and Holbrook, 2012; Gu et al., 2019; Tian et al., 2019). The internal flow problem requires appropriate reconstruction of the bounding surface that most

\footnotetext{
* Corresponding author.

Tel.: +61 399256093

E-mail address: lin.tian@rmit.edu.au

** Corresponding author.

Tel.: +1 7135009251

E-mail address: Wei-Chung.Su@uth.tmc.edu
}

realistically represent the airway geometry. For human extrathoracic airways (nasal cavity/pharynx/larynx), anatomical models were frequently rebuilt via image scans or cadaver casts (Cheng, 1999; Tu et al., 2013); however, such methodology is not practical to reconstruct human whole lung which contains millions of airway branches with terminal dimension drops to hundreds of micrometers. Instead, whole lung modeling can only be achieved through mathematical approximation guided by key indices obtained from airway samples. Weibel (1963) proposed such a whole lung model (23 generations) completely assembled by sequence of $2 \mathrm{D}$ symmetric bifurcations based on selected sampling of nonrandomly distributed structures. Mathematical equations for human lung morphometry were prescribed to quantitatively correlate the anatomy to its physiological functions. Based on the principles of: 1) volumetric proportionality between airway branches and the delivered pulmonary space; 2) homogeneous distribution of airway terminal branches, Kitaoka et al. (1999) formulated deterministic algorithms for 3D human tracheobronchial tree modeling with morphometric characteristics very similar to those obtained from airway 
cast measurements. The algorithms were further packaged into a software which can be freely downloaded for the purpose of studying lung anatomy, physiology, and pathophysiology (Kitaoka et al., 2013).

Apart from whole lung modeling, significant research was conducted in regional airways, where sectional details of the transport processes, critical to the understanding of underlying physics, can be analyzed. Focused on tracheobronchial cartilaginous rings, Martonen et al. (1994) investigated the morphology-induced variation on fluid dynamics in a simplified 2D airway passage. Using a symmetric 2D bifurcation model, Li and Ahmadi (1995) simulated the micro- and nanoparticle dispersion and deposition in human trachea and the main bronchi, where main deposition mechanisms were identified. Comer et al. (2001) performed the computational investigation of flow structures and particle deposition patterns in a doublebifurcation airway model (G3-G5). Zhou and Cheng (2005) measured the regional particle deposition efficiency and deposition patterns in a human airway cadaver replica including the oral cavity, pharynx, larynx, trachea, and four generations of bronchi. Based on a single bifurcation element (Heistracher and Hofmann, 1995; Phillips and Kaye, 1997), Tian and Ahmadi (2012) proposed a multi-level asymmetric lung bifurcation model (G0-G3), flexible to be extended, for airflow and particle transport investigation. More recently, up to the $6^{\text {th }}, 7^{\text {th }}$ and $15^{\text {th }}$ generation of anatomical tracheobronchial tree models were used by Koullapis et al. (2016), Lintermann et al. (2017), Frederix et al. (2018), Xu et al. (2019) and Dong et al. (2019a, b) for regional aerosol deposition studies. Clearly seen from these research investigations, both anatomical reconstruction and the mathematical simplified modeling approaches were engaged. While significant insights were rendered from these pioneer studies, fundamental analysis seeking morphometry-induced variations in flow pattern, particle trajectory and particle deposition statistics in human tracheobronchial airways were rare. In particular, intelligence of particle and flow partition at the airway bifurcating sites, critical to address navigated targeting delivery was not fully investigated.

To fill the gap, Shang et al. (2018) performed a numerical comparison of nanoparticle transport and deposition in human upper tracheobronchial airways (trachea and the first bifurcation truncated at five lobar partitions) by using an anatomical realistic reconstruction (through CT scans) and a mathematical simplified airway model. The investigation identified major irregularity (at head airway connection)induced variation in airflow and particle deposition patterns in the trachea, while particle fate in the bronchi were less affected. The findings correlated well with that of Zhang and Finlay (2005), though their experiments focused more on effect of cartilaginous rings and particles in the micrometer range. Accounting for this geometric irregularity at head airway connection, a modified idealized airway model (based on that of Shang et al., 2018), closer to morphometry of the anatomical construction, was developed in the current study. Using the anatomical airway model (Shang et al., 2018) as a benchmark, new boundary considerations mimicking lobar bronchi air partition were applied to the newly developed idealized model, and major trachea-irregularity-induced enhancement of nanoparticle deposition was quantified. In particular, flow and particle partitioning in the branching airways, an important postulation for aerosol distribution in whole lung dosimetry modeling (Phalen et al., 2016), was examined, and the detailed flow and particle profiles at five lobar exists were analyzed.

A paralleled experimental measurement of sodium chloride $(\mathrm{NaCl})$ nanoparticle penetration and deposition rates in the corresponding pair of anatomical and idealized airway models was developed. The computational model predictions were compared with the experimental measurement, general agreement and valuable information were obtained. It should be noted that, due to instrumentation limitation, current experiment only included nanoparticles of sizes greater than $10 \mathrm{~nm}$. However, to obtain the relationship of particle partition, deposition and penetration characteristics with respect to particle sizes, current computational simulation included a wider range of nanoparticles (1-100 nm) assuming negligible agglomeration.

Current research contributes to the understanding of morphometry sensitivity of flow and particle trajectories in human tracheobronchial airways. It is of significance in context of the increased use of tracheobronchial airway modeling in particle study in human lung. In addition, particle partitioning, an important feature for navigated delivery of nanoparticles in airway passages, was investigated. Lastly, the paralleled experiment provided valuable validation data for current and future computational studies.

\section{METHOD}

\section{Human Upper Tracheobronchial Airway Geometric Models}

An anatomical reconstruction of an adult human tracheobronchial tree up to the $15^{\text {th }}$ generation (Fig. 1(a)) was made via CT scans (Shang et al., 2018). A truncation, containing trachea and the main bronchi, was extracted and used for a morphometry comparative study with an idealized airway model developed by Shang et al. (2018). Extending from the study, a modification was made to the existing idealized model by introducing the major irregularity at head-airway connection mimicking that of the anatomical reconstruction (Fig. 1(b)). As shown in the figure, the sectional upper tracheobronchial airway terminated at entrance of the five lobes leading to deeper lung. Further details on model specification and development can be found in the work of Shang et al. (2018).

\section{Computational Mesh}

Computational mesh was generated by using Ansys Fluent Meshing (18.0), where 789,310 and 750,134 polyhedron elements of dimension $0.5 \mathrm{~mm}$ were created for the anatomical and idealized models as shown in Fig. 2. With the first grid point located $0.05 \mathrm{~mm}$ away, 10 prism layers were attached to the airway surface to resolve near-wall features. The prism layers contained hexahedron cells, growing at a rate of 1.2 from the boundary to the core region. Mesh independent study was performed on the 

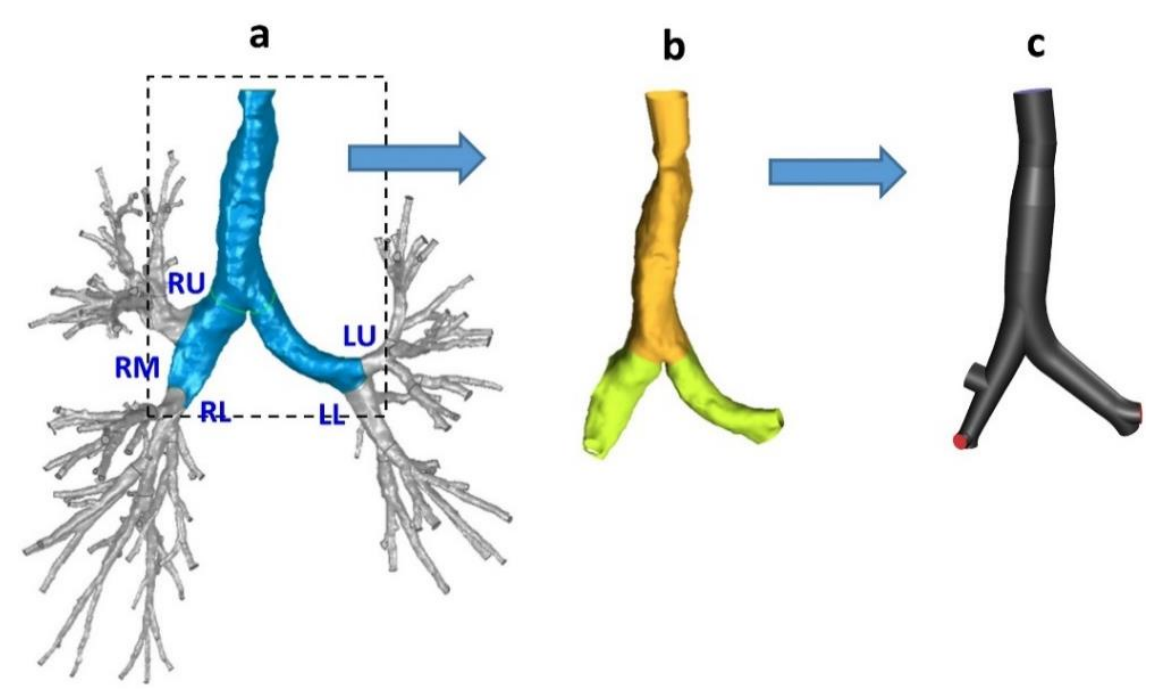

Fig. 1. Human airway geometric modeling: (a) $15^{\text {th }}$ generation anatomical tracheobronchial tree model from CT scans; (b) truncated anatomical model containing trachea and the first bifurcation; (c) mathematical idealized model.

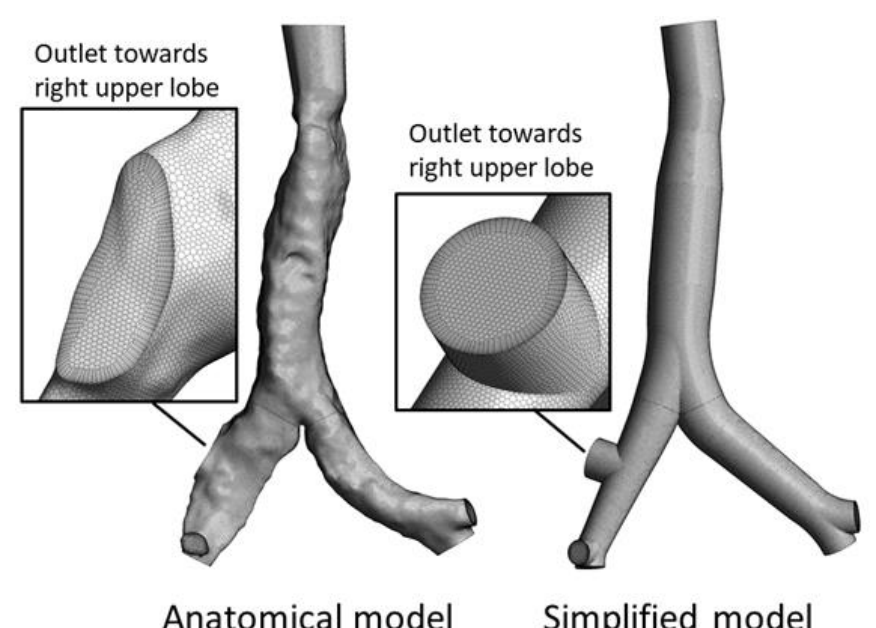

Fig. 2. Computational mesh with polyhedron meshing schemes.

newly developed idealized model, where velocity profiles along the line and across a cross section near the airway bifurcation (Fig. 3(b)) were obtained and compared by using mesh resolutions of $0.7,0.6,0.5$ and $0.4 \mathrm{~mm}$, mesh sizes of $370,588,512,786,750,134$ and 1,189,719 respectively. It was shown in Fig. 3 that velocity profiles along the selected line and cross section were insensitive to the four mesh resolutions, while Fig. 3(b) implied the optimal mesh size of 750,134 with mesh dimension of $0.5 \mathrm{~mm}$ be used for the computational study.

\section{Fluid Flow Simulation}

Steady inhalation $\left(30 \mathrm{~L} \mathrm{~min}^{-1}\right)$, assuming the particle deposition mainly occurred during inhalation phase (Inthavong et al., 2010), was employed. Breathing air was predominantly turbulent due to the presence of strong irregularities at the laryngeal (Tian and Ahmadi, 2012). Reynolds stress transport model together with the "two-layer zonal" boundary condition was used to reproduce the breathing air, as it has been shown to be the most accurate in resolving near-wall flow features, critical to micro- and nanoparticle deposition studies (Tian and Ahmadi, 2007). Details on the governing equations of turbulence mean flow, turbulence fluctuations, and turbulence near-wall modeling can be found in the work of Tian and Ahmadi (2007), and Shang et al. (2018).

Ansys Fluent 18.0 was used to perform the steady flow simulation. In physically realistic breathing cycles, airflow was driven by the pressure gradient between ambient environment and the thoracic cavity. Therefore, prescribed pressure difference between airway entrances and the exits was frequently enforced as the boundary condition in breathing airflow simulations (Koullapis et al., 2016; Shang et al., 2018). In addition to this approach, current study took an additional boundary consideration where assumed flow partition among the five lobar branches was prescribed. As the regional airway was distantly upstream the chest diaphragm where the exact pressure at each branching exists was unknown, the new boundary consideration might be more 

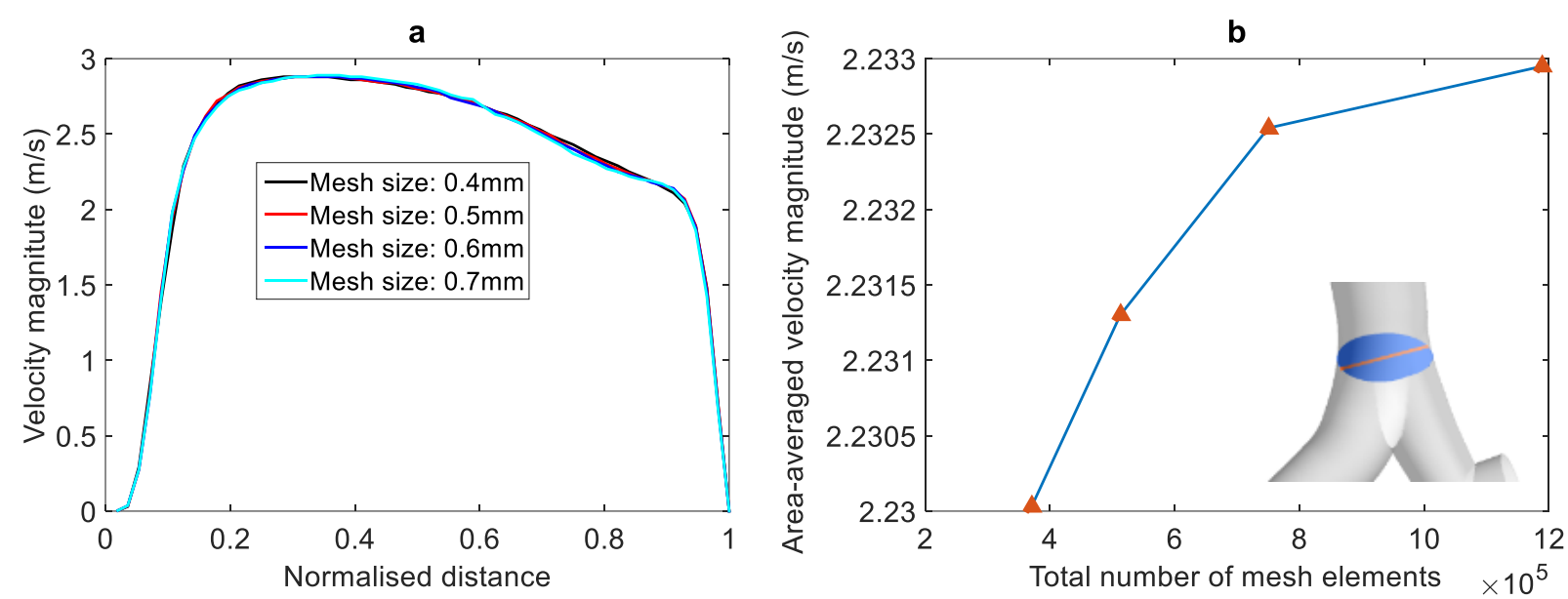

Fig. 3. Mesh independent study: (a) velocity profiles along the line of a cross section near the bifurcation; (b) area-averaged velocity magnitude on the cross section near the bifurcation.

physically appropriate. Comparisons of particle simulation results were made by using the two different boundary considerations.

\section{Particle Simulation}

Assuming particles are sufficiently dilute that the airflow field is not affected by the presence of particles, and particle-particle collision is not considered, the governing equation of motion for each particle is given as:

$$
\frac{d \boldsymbol{u}_{p}}{d t}=\frac{1}{C_{c}} \boldsymbol{F}_{\boldsymbol{D}}+\frac{\boldsymbol{g}\left(\rho_{p}-\rho\right)}{\rho_{p}}+\boldsymbol{F}_{\boldsymbol{L}}+\boldsymbol{F}_{\boldsymbol{B}},
$$

where $\boldsymbol{u}_{\boldsymbol{p}}$ is the particle velocity vector, $t$ is the time, $\boldsymbol{g}$ is the gravitational acceleration, $\rho_{p}$ and $\rho$ are the particle and fluid densities respectively. As the current study is focused on nanoparticles, gravitational and the buoyancy forces can be neglected. $\boldsymbol{F}_{\boldsymbol{D}}$ is the particle-flow slip force given by $18 \mu\left(\boldsymbol{u}_{p}\right.$ $-\boldsymbol{u}) /\left(d^{2} \rho_{p}\right)$, with $d$ being the particle diameter. In Eq. (1), $C_{c}$ is the Cunningham correction for nanoparticles which is given as:

$C_{c}=1+\frac{2 \lambda}{d}\left(1.257+0.4 e^{(-1.1 d / 2 \lambda)}\right)$,

where $\lambda$ is the gas mean free path. In Eq. (1), $\boldsymbol{F}_{L}$ is the Saffman lift force, given as:

$$
F_{L i}=\frac{2 K v^{\frac{1}{2}} d_{i j}}{S d\left(d_{l k} d_{k l}\right)^{\frac{1}{4}}}\left(u_{j}-u_{p j}\right)
$$

where $K=2.594$ is the constant coefficient (Saffman, 1965), $v$ is the fluid kinematic viscosity, $S$ is the particle-to-fluid density ratio, $u$ and $d_{i j}$ are the fluid velocity and strain-rate tensor respectively. The Brownian diffusion force $\boldsymbol{F}_{\boldsymbol{B}}$ in Eq. (1) is modeled as a Gaussian white noise random process with spectral density given by Li and Ahmadi (1992):

$$
S_{o}=\frac{216 v k_{b} T}{\pi^{2} \rho d^{5}\left(\frac{\rho_{p}}{\rho}\right)^{2} C_{c}}
$$

where $k_{b}=1.38 \times 10^{-23} \mathrm{~J} \mathrm{~K}^{-1}$ is the Boltzmann constant and $T$ is the absolute temperature.

Particle simulation was performed by using the discrete phase model (DPM) of Ansys Fluent 18.0. 10,000 monodispersed ultrafine particles from 1 to $100 \mathrm{~nm}$ were uniformly released from the trachea entrance. Particle trajectories, deposition rate and penetration statistics were calculated. It was assumed that particle deposited onto the airway surface upon touching.

\section{Deposition Intensity Factor}

To quantify particle deposition patterns in the tracheobronchial airway surface, the deposition intensity factor (DIF) is defined as the ratio of the number of deposited particles onto $1 \mathrm{~mm}^{2}$ surface area of the local region to the total number of released particles:

$D I F=\frac{\# \text { reginal deposited particles } / \mathrm{mm}^{2}}{\# \text { particles entering the computational domain }}$

The parameter is similar to a prior defined deposition enhancement factor (DE), formulated as the regional particle deposition within a defined area relative to the total deposition normalized by the surface area of the entire region (Balásházye et al., 1999; Zhang et al., 2005; Longest and Holbrook, 2012). The DIF is continuously defined across all surface area of the tracheobronchial airway model, where particle deposition density can be easily visualized. The choice of $1 \mathrm{~mm}^{2}$ as the local region is determined by the optimal visual representation of the particle deposition results in the current study. 


\section{Experimental Setup}

In this study, lung deposition experiments were conducted by delivering sodium chloride nanoparticles $(10,20,50$, and $100 \mathrm{~nm}$ ) into the physical models of the airway (anatomical model and the mathematical idealized Model) (Fig. 4). The airway physical replicas were printed using a highresolution 3D printer (LulzBot TAZ 6; Aleph Objects, Inc., Loveland, Colorado, USA) with conductive PLA filaments (Proto-pasta; ProtoPlant, Vancouver, WA, USA). By obtaining the penetration efficiency of the $\mathrm{NaCl}$ particles for all lung tubes on the airway replica, $\mathrm{NaCl}$ particle deposition fraction (\%) in a certain airway bifurcation can be calculated. The experimental approach has been used previously to estimate lung deposition of airborne carbon nanotubes and graphene (Su and Cheng, 2015; Su et al., 2016). To facilitate the measurement of $\mathrm{NaCl}$ particle penetration efficiencies at a certain lung tube on the airway replica, a set of trimmed airway replicas were made similar to those shown in $\mathrm{Su}$ and Cheng (2015). Prior to the experiment, the surface of the airway replica was coated with a thin layer of silicon oil to prevent the resuspension of the deposited particles. To generate the nanoscaled $\mathrm{NaCl}$ particles $(10,20,50$, and $100 \mathrm{~nm}$ ), an atomizer aerosol generator (3079A; TSI Inc., Shoreview, MN, USA) was employed using sodium chloride water solution $(0.5 \% \mathrm{v} / \mathrm{v}) . \mathrm{NaCl}$ aerosols generated by the atomizer were immediately delivered to a series of drying columns to allow water mist in the $\mathrm{NaCl}$ aerosols to completely evaporate. Then, nanoscaled $\mathrm{NaCl}$ particles were charge neutralized and delivered into the airway replicas (untrimmed and trimmed airway replicas) for deposition measurement. It should be noted that, due to current technical limitation, generation and classification of $\mathrm{NaCl}$ nanoparticles below $10 \mathrm{~nm}$ is not possible.

Two units of nanoparticle sizer (SMPS+C; GRIMM Aerosol Technology, Germany) were used simultaneously, with one unit connected to the trachea inlet, and the second unit connected to the outlet of the specified lung tube along the airway replica. The two particle sizers were set at the same particle size channel to simultaneously measure the number concentration of $\mathrm{NaCl}$ particles at the specified size. Consequently, penetration efficiency at a specific nanoparticle size for a certain lung tube can be obtained. Measurements of $\mathrm{NaCl}$ particle penetration efficiency were carried out in turn for all lung tubes along the airway replica. Fig. 4 shows the experimental setup of nanoparticle lung deposition experiments. When penetration efficiencies of all lung tubes on the airway replica were acquired, the deposition fraction of the nanoscaled $\mathrm{NaCl}$ particles in each airway bifurcation can be reasonably calculated given air flow rate in each lung tube. Details of the approach (methods and equations) can be found in the work of Su and Cheng (2015) and Su et al. (2016).

\section{RESULTS AND DISCUSSION}

\section{Breathing Airflow Pattern}

Streamwise airflow in the anatomical and newly developed idealized airway models were displayed in Fig. 5. Main features of the pressure and velocity distribution conformed to each other in the two representations. Both captured the velocity acceleration and pressure drop in vicinity of the head airway connection due to bending and contraction. Airway contraction was more radical in the anatomical model that led to a higher pressure drop, flow acceleration and larger flow recirculation zone behind the bend. At carina ridge, elevated pressure was observed in both the anatomical and idealized models, corresponding to the flow stagnation region where partitioning of the breathing air took place before entering into the daughter branches. It was observed in Fig. 5 that bulk air penetrated into the inner walls and was more pronounced in the daughter branch with sharper branching angle (left bronchi). Higher level of mixing of the breathing air was observed in daughter branches of the anatomical model than that in the idealized one.

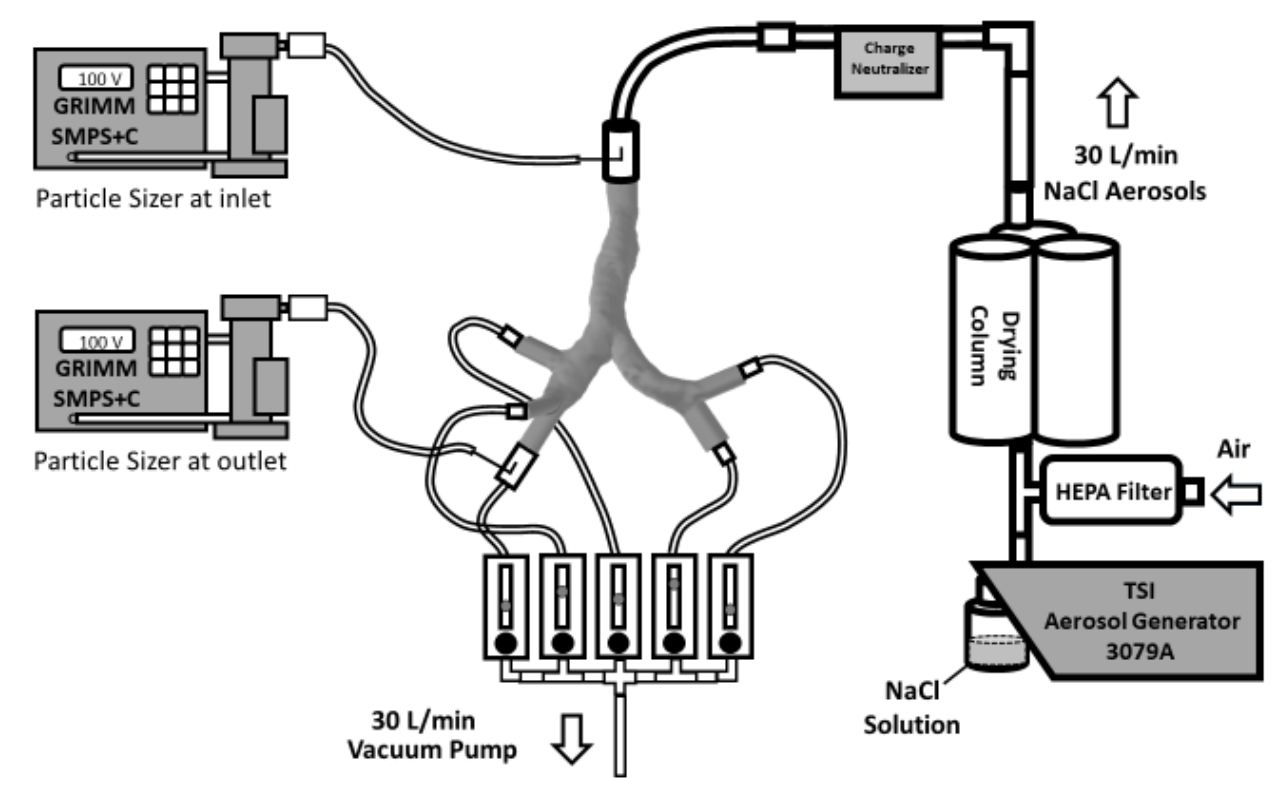

Fig. 4. The experimental setup of nanoparticle lung deposition measurements. 


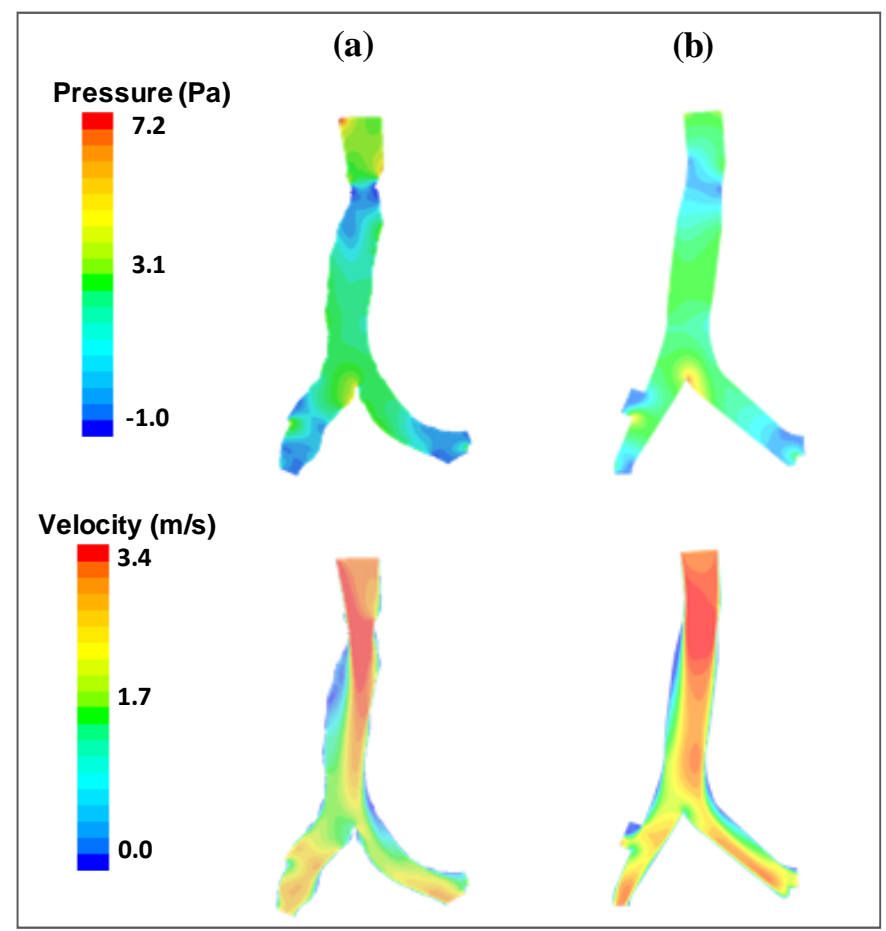

Fig. 5. Streamwise airflow patterns (pressure and velocity magnitude) at breathing rate of $30 \mathrm{~L} \mathrm{~min}^{-1}$ in the central plane of the tracheobronchial airways: (a) truncated anatomical model; (b) mathematical idealized model.

Velocity vectors of the secondary flow at selected cross sections along the airway was shown in Fig. 6. Due to space limitation, selected sample locations were only demonstrated on the anatomical model in the figure. It was noted that the color map includes the streamwise velocity component, but length of the vectors only account in plane secondary-flow components. Corresponding to the observation in Fig. 5, level of the secondary flow in the anatomical model was relatively higher than that in the idealized representation throughout the airways. It was seen from Fig. 6 that secondary flow was mainly induced by the abrupt changes of airway morphology (e.g., bend, contraction, and the bifurcation), while effect of the boundary layer formation was insignificant. Higher level of mixing in the anatomical model, reflected by the more uniform secondary-flow distribution, might be attributed to the wavy wall roughness in the trachea due to cartilage rings and the deformation of airway passage from circular to highly oblate cross-sectional areas at transition from trachea to the daughter branches.

\section{Turbulence}

Fig. 7 compared the turbulent kinetic energy at the breathing rate of $30 \mathrm{~L} \mathrm{~min}^{-1}$ in the anatomical and newly developed idealized airway models. Significant turbulence production was observed at the trachea contraction in the anatomical model (Fig. 7(a)), where turbulence filled the entire region up to the carina ridge. A secondary production, at much smaller scale, was induced by flow partition immediately following the bifurcation. In the main channels (trachea and daughter branches), turbulence was continuously dissipated as the airflow traveled downstream (Fig. 7(a)). When looking at the idealized model (Fig. 7(b)), main feature of the turbulence activity was captured, however to a lesser degree where trachea contraction was introduced near the entrance. The generated turbulence was seen to propagate to a smaller region, and dissipated more quickly than that in the anatomical model. In both models, left bronchi hosted slightly higher-level turbulence activities than that in the right bronchi.

\section{Wall Shear Stress}

Fig. 8 displayed the wall shear stress of the airway surfaces mapping to a 2D domain for superior visualization. Details on surface mapping technique and the applications can be found in the work of Inthavong et al. (2014), Tian et al. (2017a, b), and Shang et al. (2018). As shown in the figure, global high-shear zones agreed in both models, which were at the trachea entrance and in the vicinity of the airway bifurcations. Due to bending and contraction near trachea entrance, both models experienced high level of shear stress in upper sections of the airways (Figs. 8(a) and 8(b)). Significant difference between the anatomical and idealized models was the small-scale local wall shear fluctuation, which was observed throughout the anatomical airway surface (Fig. 8(a)), however, not seen in the idealized model (Fig. 8(b)). Very low and uniform distribution of the wall shear stress in the trachea and bulk region of the daughter branches in the idealized model (Fig. 8(b)) implied a smooth airway surface with low friction. On the contrary, sporadic and streak-patterned wall shear stress in the anatomical model (Fig. 8(a)) implied a rough surface corresponding to the cartilage rings and predominantly wavy interior wall. Wall shear distribution was closely related to the formation of near-wall eddies that contribute to particle deposition processes. 


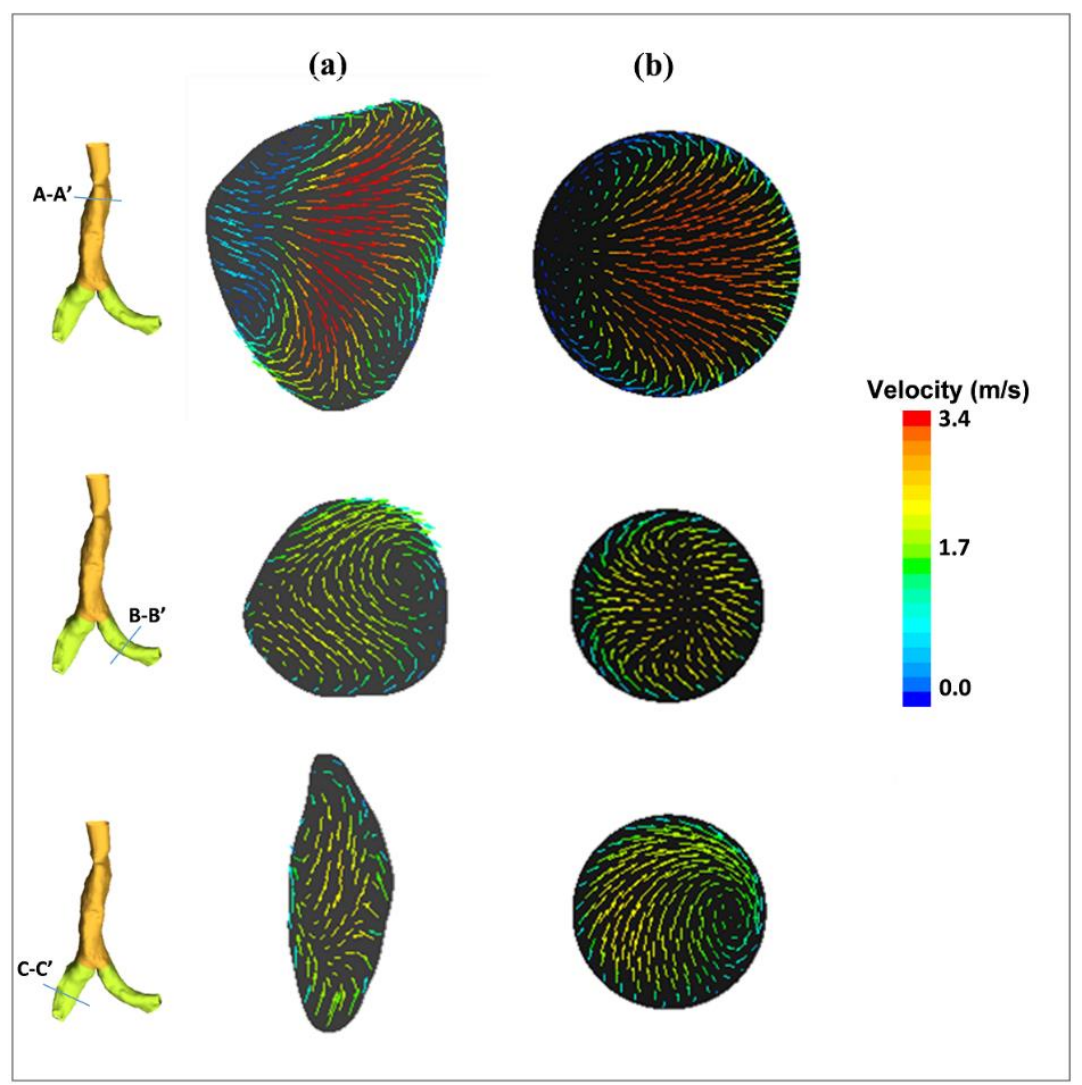

Fig. 6. Secondary flow pattern at cross sections along the trachea, left and right bronchi: (a) anatomical model; (b) idealized model.

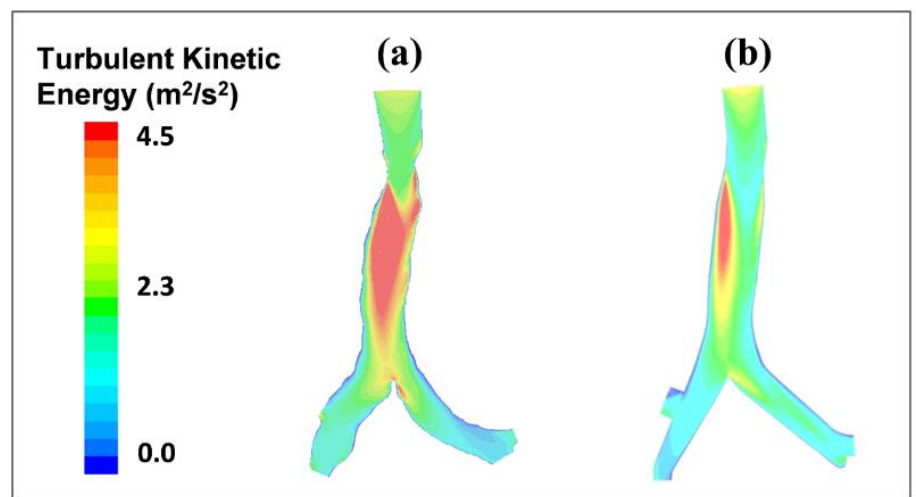

Fig. 7. Turbulence kinetic energy at breathing rate of $30 \mathrm{~L} \mathrm{~min}^{-1}$ in central plane of the tracheobronchial airways: (a) truncated anatomical model; (b) mathematical idealized model.

\section{Particle Deposition Pattern}

To visualize particle deposition pattern, DIF (Eq. ((5)) of 10,000 uniformly released monodispersed unit density solid particles (1 and $100 \mathrm{~nm}$ ) were calculated and presented in Fig. 9. DIF quantified the local particle deposition density, and facilitated the identification of deposition hot spot and variations in the domain. For the $1 \mathrm{~nm}$ particles, deposition pattern in the newly developed idealized model (Fig. 9(b)) was similar to that in the anatomical model (Fig. 9(a)), where elevated deposition was observed sporadically in vicinity of the bending near trachea entrance. However, the deposition "hot region" in the anatomical model exhibited higher intensity and covered a larger area. For the $100 \mathrm{~nm}$ particles, neither the anatomical nor idealized model displayed deposition enhancement induced by the trachea bending, however, the slightly higher deposition throughout the airway surface as seen in Fig. 9(a) could be attributed to the local wall roughness (e.g., cartilage rings) in the anatomical model. It was clearly shown in Fig. 9 that regional deposition of nanoparticles in the upper tracheobronchial airways was size dependent. $1 \mathrm{~nm}$ particles were more likely to be deposited in the trachea, while significant fewer deposited in the main bronchi. For the $100 \mathrm{~nm}$ particles, no distinctive deposition preference was observed in both models, while slight higher 


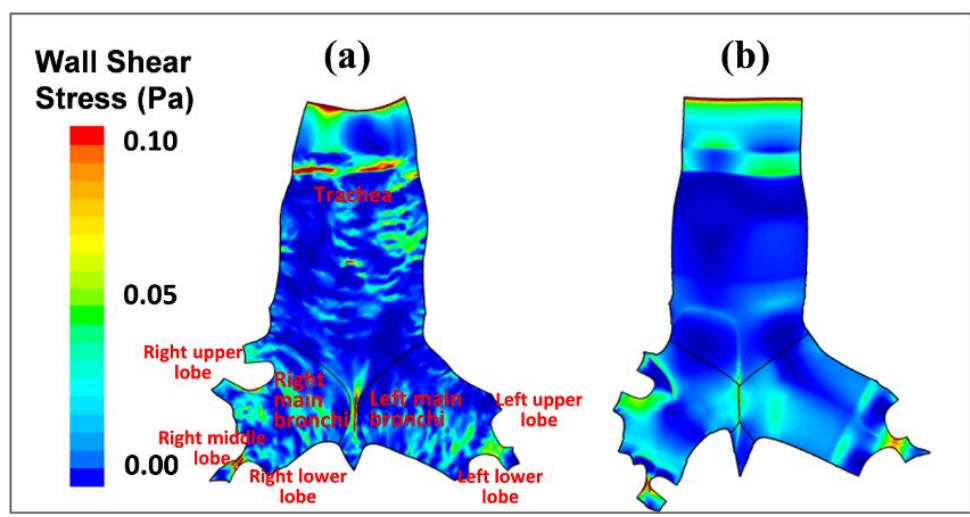

Fig. 8. Wall shear stress on the unwrapped 2D surface models of the tracheobronchial airways: (a) truncated anatomical model; (b) mathematical idealized model.

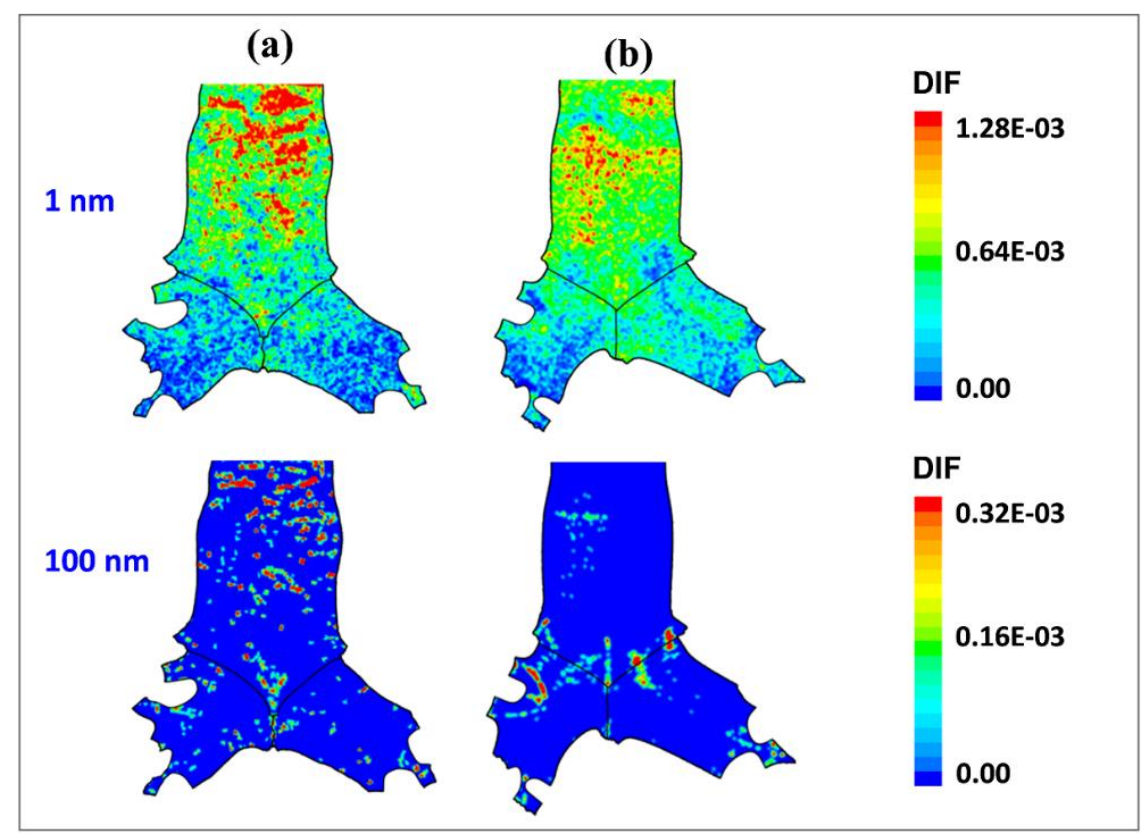

Fig. 9. Particle deposition intensity factor (DIF) for 1 and $100 \mathrm{~nm}$ particles in the 2D unwrapped surface models: (a) truncated anatomical model; (b) mathematical idealized model.

deposition intensity was seen near the bifurcation ridge, especially in the idealized model (Fig. 9(b)). Still, slight higher level of deposition of $100 \mathrm{~nm}$ particles in the trachea was observed in the anatomical model than that in the idealized model.

Finally, a comparison between Figs. 8 and 9 showed a remarkable resemblance between the airway wall shear stress and particle DIF for the $100 \mathrm{~nm}$ particles. This was not seen with the $1 \mathrm{~nm}$ particles, indicating the presence of a molecular-diffusivity-dominated transport process other than a transport mechanism dictated by the particle-flow inertia interaction as seen in the earlier case.

\section{Particle Penetration Profile}

Figs. 10 and 11 displayed the penetrating profiles of 1 and $10 \mathrm{~nm}$ particles at the five bronchi exits before entering into deeper lung. For both sized particles, penetrating profiles varied in the anatomical and idealized models. In general, improved mixing of the inhaled nanoparticles was observed in the anatomical model, where particles were seen to disperse to all regions at the cross sections though preferential concentration might be exhibited. This was not observed in the idealized model (Figs. 10(b) and 11(b)), where all cross sections had space void of inhaled nanoparticles. In particular, large void space, free from particles, was clearly seen in the right middle (RM) and right upper (RU) exits.

A unique vortex-like penetrating profile was observed for the $10 \mathrm{~nm}$ particles as they exited from the left upper (LU) branch in both models (Fig. 11). This indicated the evolution of a vortex-like secondary flow which lead to the left upper lobe. An interesting observation was that the $1 \mathrm{~nm}$ particles did not respond to this unique secondary-flow pattern and particle distribution remained random across the LU exits (Fig. 10). It was also noted that level of inhomogeneity in particle distribution was increased when particle size grew from 1 to $10 \mathrm{~nm}$ (Figs. 10 and 11). Apparently, particles 


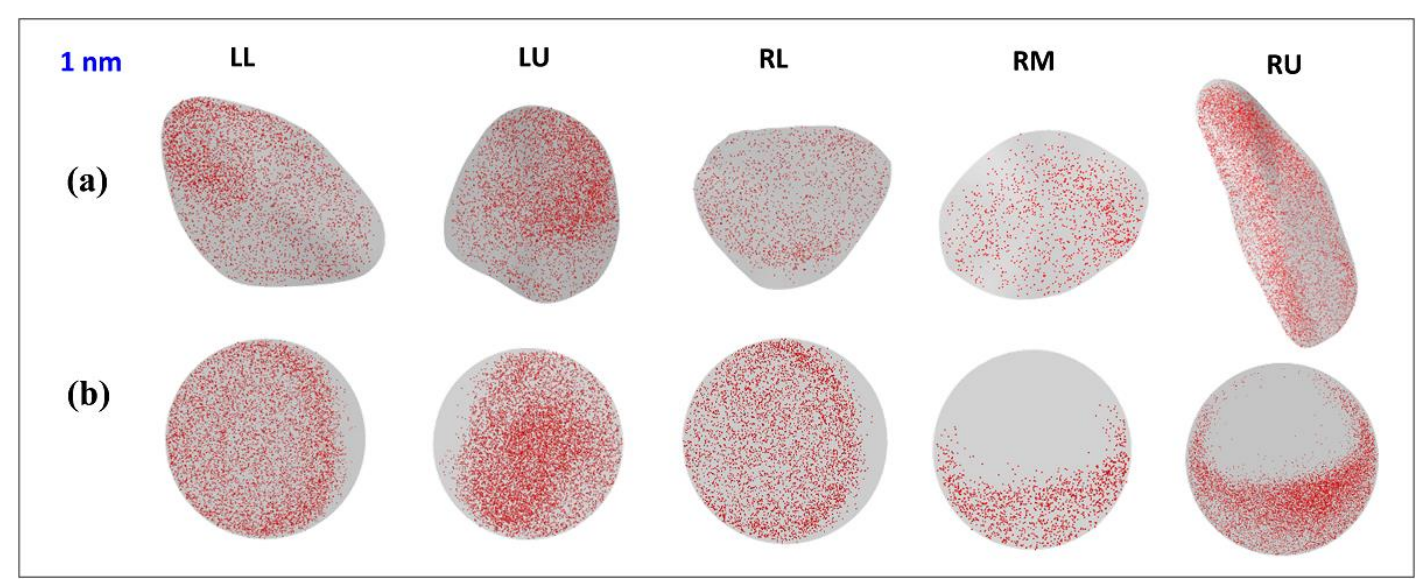

Fig. 10. Particle distribution (1 nm) at 5 bronchi exits (LL: left lower lobe; LU: left upper lobe; RL: right lower lobe; RM: right middle lobe; RU: right upper lobe): (a) truncated anatomical model; (b) mathematical idealized model.

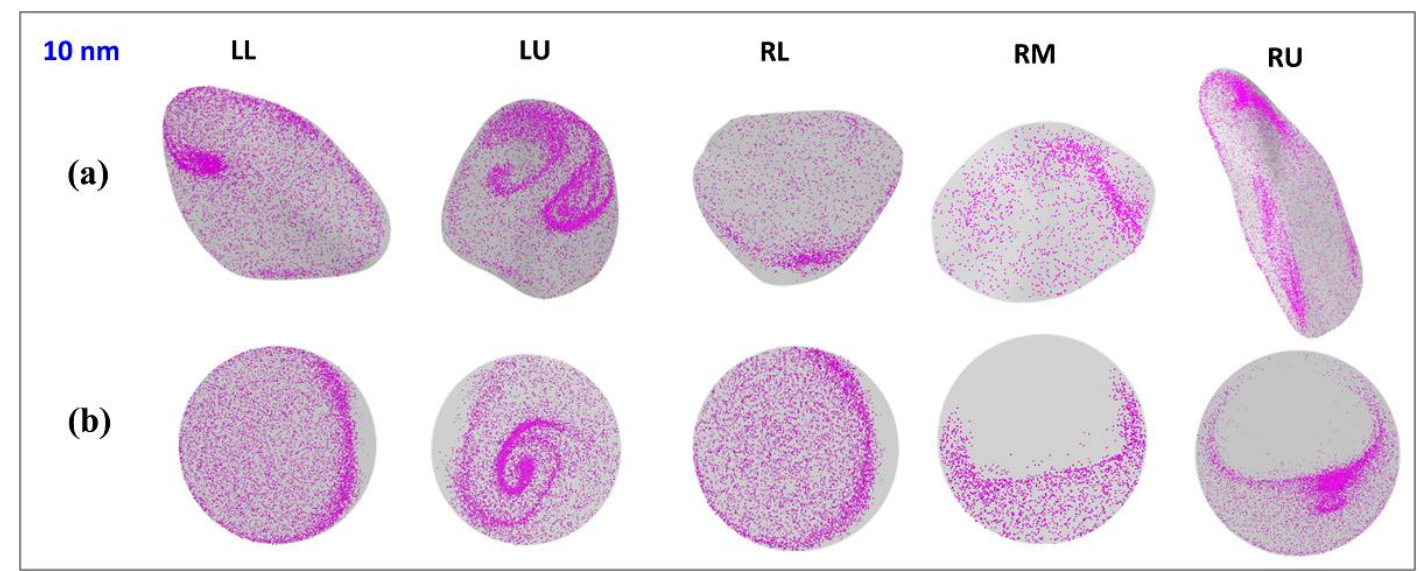

Fig. 11. Particle distribution ( $10 \mathrm{~nm}$ ) at 5 bronchi exits (LL: left lower lobe; LU: left upper lobe; RL: right lower lobe; RM: right middle lobe; RU: right upper lobe): (a) truncated anatomical model; (b) mathematical idealized model.

responded more to the flow with increased particle inertia, and preferential particle trajectories were starting to be formed. It was also noted that the largest discrepancies (in particle penetrating profile) between the idealized and anatomical models were seen in the RM and RU branches.

\section{Particle Deposition Efficiency}

Fig. 12 displayed the total and regional deposition efficiency of the nanoparticles entering from trachea of the tracheobronchial airway models. Particle deposition efficiency is defined as the ratio of the deposited particles in a region to the total number entering the domain. To gain further insight of particle-flow relationship, one more set of simulation were performed in the idealized model, with new boundary condition assuming prescribed flow partition in the five lobes following that of the anatomical model (Table 1). Simulation results from Shang et al. (2018), by using a prior developed idealized model without accounting for the geometric irregularity at head-airway connection, was also included for comparison. Experimental measurement on nanoparticle deposition in human tracheobronchial airway replicas in current and prior studies were plotted along with the computational simulations. It was shown in Fig. 12(a) that the simulated nanoparticle total deposition efficiency was the highest in the anatomical model (red -o-). By accounting for the geometric irregularity at head-airway connection, particle depositions in the newly developed idealized model (blue - $\square-,-\diamond-$ ) were very close to that in the anatomical model. This was a significant improvement from simulation predictions without accounting for this geometric irregularity (green -*-, - - $\boldsymbol{\zeta}_{-}$; Shang et al., 2018). However, slightly lower deposition was still seen in the newly idealized model (Fig. 12(a)), which could be attributed to the added deposition due to small-scale surface roughness in the anatomical model (Fig. 1).

A subtle yet important transition occurred at about $20 \mathrm{~nm}$ (Fig. 12(a)), where the deposition curve of the new idealized model deviated from that of the anatomical model, and approached to that of the prior idealized model (Shang et al., 2018), when particle size continued grew. This implied that, for larger nanoparticles $(\mathrm{d}>20 \mathrm{~nm})$, deposition was less sensitive to macroscale irregularities, however more affected by the small-scale surface roughness. The enhancement of particle deposition due to bending and contracting near the tracheal entrance gradually phased out for larger particles $(\mathrm{d}>20 \mathrm{~nm})$. Another interesting observation in Fig. 12(a) 
(a) Total Deposition

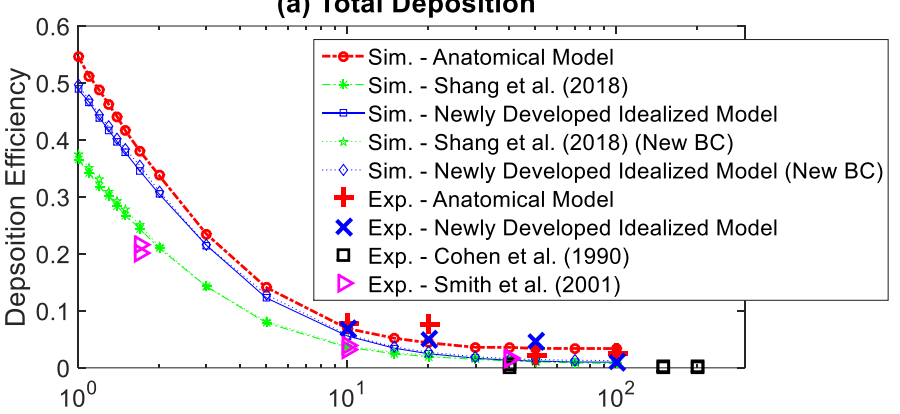

(c) Left bronchi

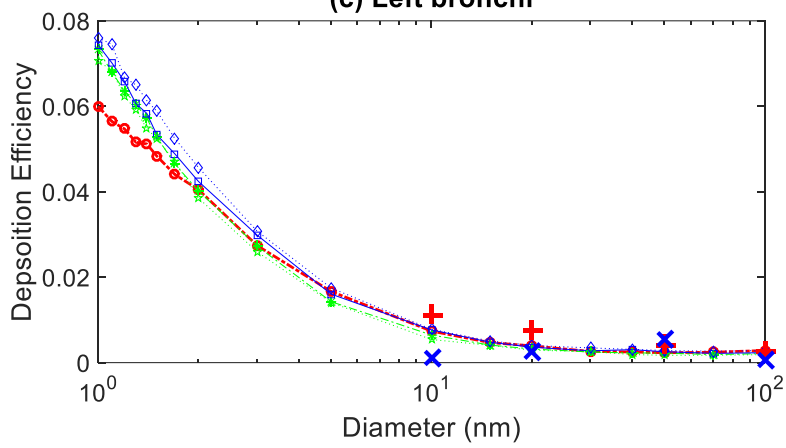

(b) Trachea

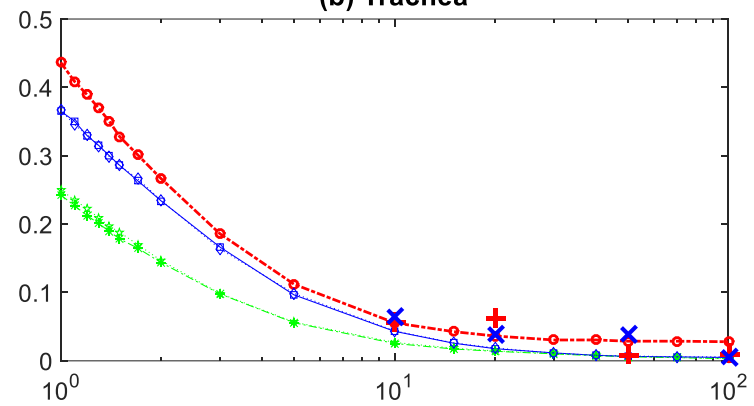

(d) Right bronchi

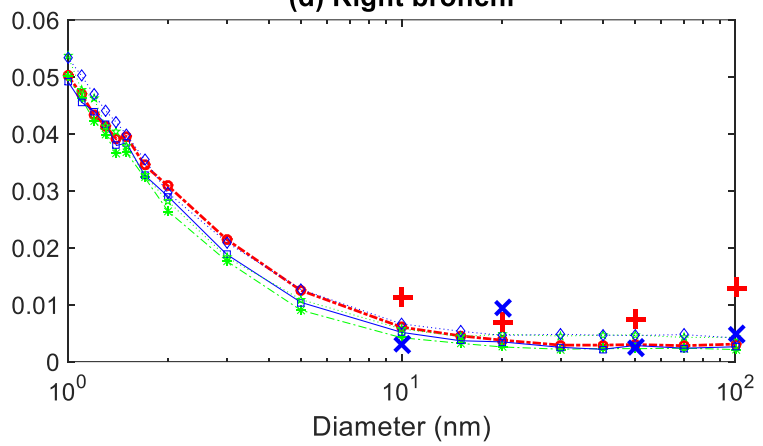

Fig. 12. Total and regional particle deposition efficiency in the airway models: (a) total deposition; (b) deposition in the trachea; (c) deposition in the left bronchi; (d) deposition in the right bronchi.

Table 1. Flow partition in the anatomical airway model.

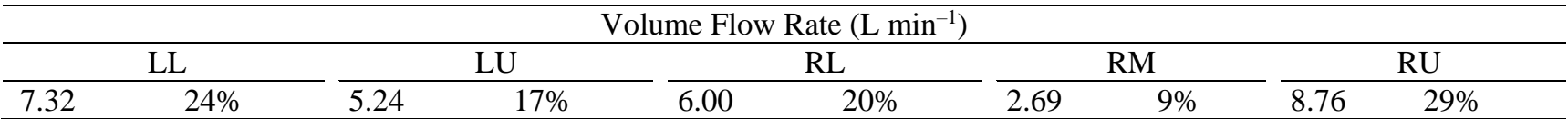

that total deposition in the truncated airways was insensitive to the prescribed boundary conditions (pressure driven versus prescribed flow partitioning among five lobes). Deposition was mainly governed by the global structure of the geometry and airflow.

Finally, the simulation agreed well with both current and prior experimental data in trend and magnitude. In particular, the measured total deposition efficiency in the anatomical reconstruction (Fig. 12(a), red +) was generally (except at $50 \mathrm{~nm}$ ) slightly higher than that in the measurement in the idealized model (blue $\mathrm{x}$ ), which supported the simulation results. It should be noted that the slight higher prediction of the deposition in current simulation and the experiment than that in the prior experiments (Cohen et al., 1990; Smith et al., 2001), was most likely to be caused by inter-subject variations in the two studies. Reported experimental data on nanoparticle deposition were extremely limited for size smaller than $10 \mathrm{~nm}$. Current experimental capability also prohibited the characterization and measurement of nanoparticles smaller than $10 \mathrm{~nm}$. Further experimental research in extreme submicrometer particle sizes $(<10 \mathrm{~nm})$ are greatly in need.

Deposition efficiencies in the trachea (Fig. 12(b)) were similar to that of the total deposition in tracheobronchial airway models. Anatomical model captured the most inhaled nanoparticles, idealized model without accounting for the irregularity at head-airway connection (Shang et al., 2018) captured the least and the newly developed idealized model was in between. Key observations, such as deposition insensitivity to boundary conditions (pressure driven versus prescribed flow partitioning among five lobes) and the transition of deposition mechanisms at about $20 \mathrm{~nm}$, were reproduced in Fig. 12(b). Nanoparticle deposition in the left and right bronchi was significantly lower than that in the trachea (5-8\% versus $25-45 \%$ ), and deposition efficiency was about the same in both the anatomical and idealized models (Figs. 12(c) and 12(d)). It should be noted that the lower level of deposition observed in the left bronchi of the anatomical model for $\mathrm{d}<3 \mathrm{~nm}$ was most likely caused by fewer particles entering the branch due to high deposition in prior pathway (trachea). Fig. 12(c) and 12(d) indicated that the geometric variation at the trachea entrance had negligible effect towards nanoparticle deposition downstream in the first-level bronchi. Once again, current experimental measurement agreed well with the simulation in trend and magnitude in regional depositions in the trachea, left and right bronchi (Figs. 12(b), 12(c) and 12(d)). A consistently slight higher deposition in the bronchi (Figs. 12(c) and 12(d)) was measured in the anatomical replica than that in the replica of the newly developed idealized model, however, this was not perceived in the trachea (Figs. 12(b)). 


\section{Particle and Flow Partition in the Main Bronchi and the Five Lobar Branches}

Fig. 13 examined the particle and flow partition (\% distribution) in the main bronchi. To gain further insight of particle-flow partitioning relationship, one more set of simulation were performed in the idealized model of Shang et al. (2018) and newly developed idealized model (Figs. 13(d) and 13(e)), with new boundary condition assuming prescribed flow partition in the five lobes following that of the anatomical model (Table 1). Fig. 13 were plotted on the same scale for optimal comparison. Clearly seen in Figs. 13(b) and 13(c) that flow driven by the pressure gradient in the idealized models incurred different flow partitioning when compared to that in the anatomical model (Fig. 13(a)). It was also noted from Fig. 13 that, even with prescribed volume flow rate following the flow partitioning in the anatomical model, particle partitioning in all configurations was distinctive in magnitude as well as in patterns. Striking match between flow and particle partitioning was observed in Fig. 13(e) (newly developed idealized model with new flow boundary condition assuming prescribed flow partition in five lobes), however, it was not reproduced in any other configurations. In general, flow and particle partitioning in the main bronchi was not necessary in proportion, and percentage distribution of nanoparticles in the two bronchi was size dependent up to $10 \mathrm{~nm}$ (except for the newly developed idealized model with new boundary condition; Fig. 13(e)) and then it approached a steady state $(10-100 \mathrm{~nm})$. Nanoparticle partitioning in the two main bronchi mostly varied between 4 to $10 \%$, and in the extreme case of $20 \%$ (Fig. 13(e)). Least variation or equal particle partitioning was observed in the anatomical model and in the pressure-driven newly developed idealized model (Figs. 13(a) and 13(c)).

Experimental measurement of particle partition in the two main bronchi, at the specified branching volume flow rate (Table 1), was presented in Figs. 13(a) and 13(e). For the size of particles considered $(10,20,50$ and $100 \mathrm{~nm})$, particle partition was seen to closely resemble that of the flow in both anatomical and the newly developed idealized airway replicas. The measured particle partition highly agreed with simulation results in the newly developed idealized model (assuming prescribed flow rate in five lobes; Table 1, Fig. 13(e)); however, measured partition in the anatomical replica did not agree with the simulation (Fig. 13(a)), where particles were almost equally parted into the two bronchi. The discrepancies might be related to the inhomogeneous particle penetration profiles (Figs. 10 and 11), which were not accounted for in the experiment with only one single reading of particle number concentration at each lung tube outlet. The discrepancy in Fig. 13(a) could also be related to boundary conditions, where the simulation was initiated by pressure difference while enforced flow partitioning was applied in the experiment. It was clearly shown in Fig. 13 that particle partition was most likely affected by both flow partitioning and particle penetration profiles. Fig. 13 also implied the sensitivity of particle partition to boundary conditions in the simulation. Further research is needed, in both the computational and experimental domains, to identify the most physical realistic boundary condition to be
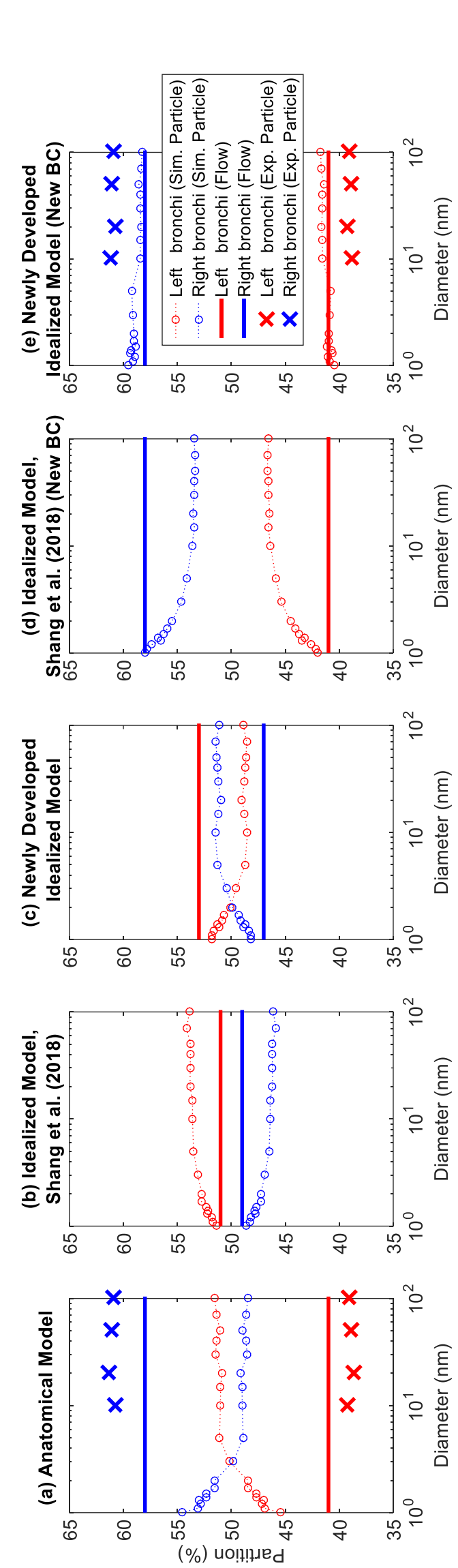

응 
applied in particle partition study to recover the realistic transport processes.

Fig. 14 displayed the particle and flow partition (\% distribution) in the five lobar branches. Similar to Figs. 12 and 13 , one more set of simulation were performed in the idealized model of Shang et al. (2018) and newly developed idealized model (Figs. 14(d) and 14(e)), with new boundary condition assuming prescribed flow partition in the five lobes (Table 1). All subplots in Fig. 14 were on the same scale for optimal comparison. Clearly seen in Fig. 14, both the flow and particle partitioning in the RM branch was the lowest $(5-10 \%)$ among all configurations. While flow partition in LU was the next lowest ( 15-20\%), particle partition in LU was relatively high in all simulations. In all configurations, particle partition in LU was over $25 \%$. Agreeable trend of the particle and flow partition was also observed in the RU branch, where the highest percentage of the particle penetration correlated to the highest volume flow rate (Figs. 14(a), 14(d) and 14(e)). However, it should be noted that with pressure gradient boundary condition (Figs. 14(b) and 14(c)), volume flow rate in the RU branch was about $20 \%$, roughly $10 \%$ lower than that in the anatomical model, yet particle partition was still among the highest in all branches. While particle partition in the left lower (LL) and right lower (RL) branches were among the lowest $(<20 \%)$, the volume flow rate was in the medium range in the anatomical model or the highest in the idealized models with pressure gradient boundary condition.

Particle partition in five lobar branches were experimentally measured in the anatomical model and the newly developed idealized model (Figs. 14(a) and 14(e)). The experimental data were straightforward that all partition followed exactly the flow distribution with only minor deviations. The measurement supported simulation observation that the highest and lowest partition occurred in the RU and RM lobes respectively. However, simulated particle partition in other lobar branches were not in proportion to flow distribution as what was observed in the experiment. As pointed out earlier, with only one reading of the particle number concentration at each lung tube outlet, rather than accounting for the inhomogeneous particle penetrating profile as shown in Figs. 10 and 11, might attribute to the discrepancy between experiment and the simulation.

Important derivations from simulation results (Fig. 14) were:

- Flow partition among five lobar airways was dependent on airway boundary conditions. Flow driven by pressure gradient or flow driven by prescribed volume flow rate could yield different flow partition in subsequent airway branches.

- Particle partition was relative consistent in the five lobar airways. Independent of geometric modeling and boundary consideration in current study, the highest to lowest particle partition in the five lobes was RU, LU, LL, RL, and RM, respectively.

- Upper lobar branches (RU and LU) attracted significantly higher portion $(>70 \%)$ of the inhaled nanoparticles into deeper airways.

- Minimum nanoparticles $(\leq 5 \%)$ passed through the RM (right middle) branch.

- Flow and particle partition in lung lobes were not necessary in proportion. Flow partitioning information cannot be used to predict nanoparticle partition in lung lobes.

- Partitioning of nanoparticles in the five lung lobes were size dependent up to $10 \mathrm{~nm}$. Beyond that, partitioning was steady and size independent.

Important derivations from experiment (Fig. 14) were:

- Particle partition among five lobar airways closely follow flow distribution and independent of geometric models.

In summary, particle partition among lung lobes and the downstream branches contains valuable information for the design of active controlled medical devices for particle delivery in human respiratory systems. Further research is needed to identify a clear relationship between particle and flow partitioning in respiratory airways. The effect of inhomogeneity of flow and particle distribution along lung airways towards particle partition transport needs further investigation.

\section{CONCLUSIONS}

This study employed an anatomically realistic model of a human tracheobronchial airway as well as a newly developed idealized extension including the trachea and two main bronchi to investigate the transport and partitioning of nanoparticles and the influence of airway morphology. Experimental measurements were also conducted in parallel. Based on our results, we drew the following conclusions:

- To accurately simulate the transport of nanoparticles (d $<20 \mathrm{~nm}$ ) in the human trachea, the major geometric features of the airway must be incorporated, although those of the secondary bronchi exert less influence.

- Local small-scale wall shear fluctuations were observed across the surface of the airway in the anatomical model but not the idealized model; this phenomenon may be related to the higher deposition efficiency of particles in the former, which was especially prominent for the fraction larger than $20 \mathrm{~nm}$. The physical mechanisms underlying the relationship between small-scale superficial roughness and particle deposition in the human airway require further investigation.

- The anatomical model exhibited lower shear stress and lower deposition at the bifurcation site (for larger nanoparticles), suggesting that transport is more efficient in the anatomical design than the idealized. Further investigation is necessary to determine whether this difference is related to the extreme irregularity of the airway geometry.

- While traveling downstream, the nanoparticles were far more thoroughly mixed in the anatomical model than the idealized model. The effect of physical properties that are associated with this phenomenon, such as the airway geometry, small-scale superficial roughness of the channel, and bifurcation structure, requires further investigation.

- A stronger response to the local flow patterns of larger nanoparticles in certain regions, such as the upper left bronchial exit, which displayed vortex-like nanoparticle 

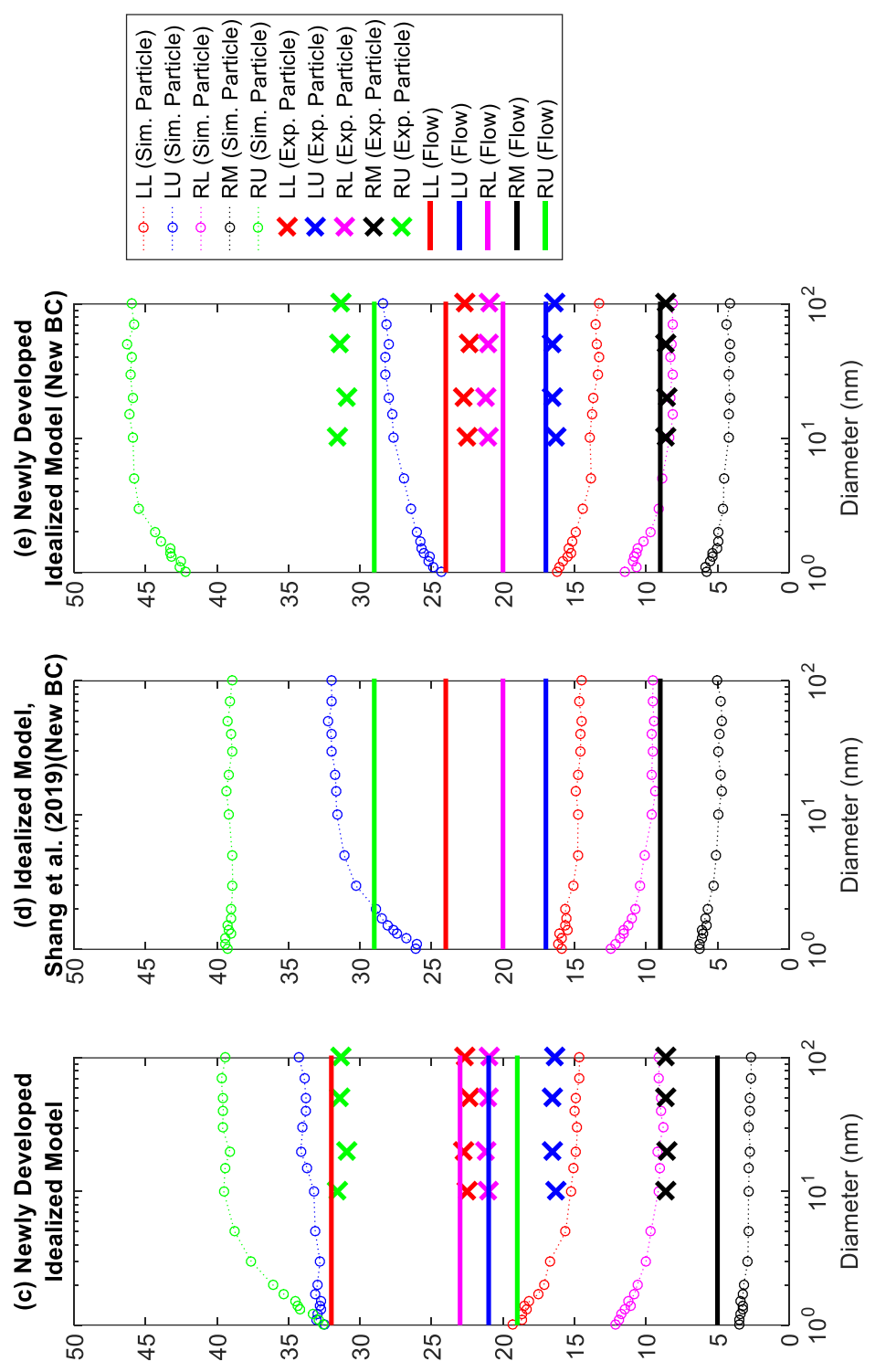

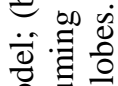

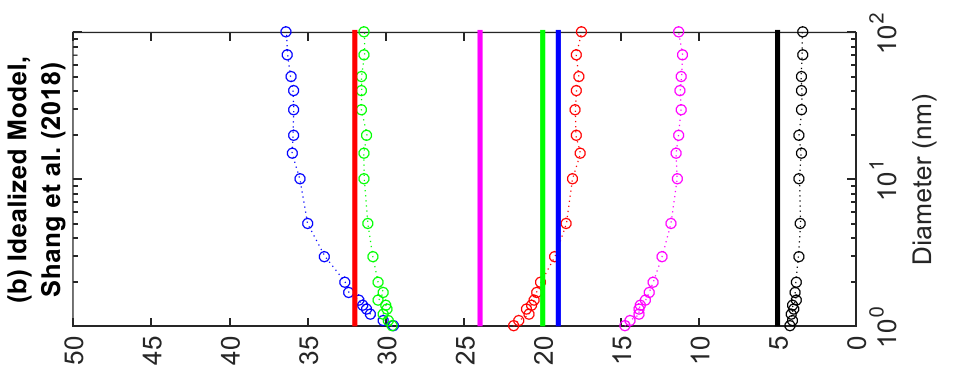

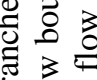

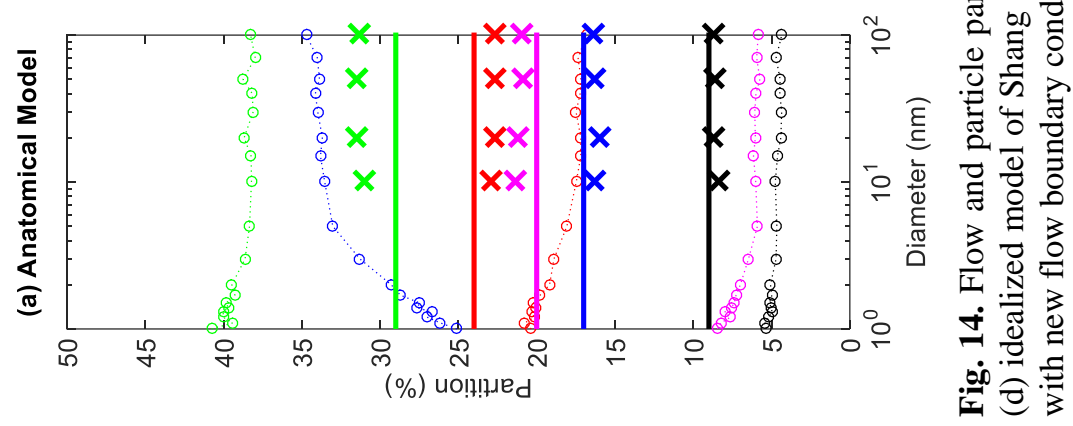


penetration, may shed light on enhancing the efficiency of targeted delivery systems.

- Contrary to the experimental results, the particle and flow partitions in the lung lobes were not necessarily proportional in the numerical simulation. A clear relationship between these partitions must be established through additional research before using flow partitioning data to predict nanoparticle partitioning in this region.

- In the simulation, the flow partitioning in the five lobar airways was dependent on the boundary conditions, whereas the particle partitioning was relatively consistent, regardless of the boundary conditions or modeled geometry. The largest particle partition was found in the upper right lobe, followed by the upper left, lower left, lower right, and center right lobes.

- The nanoparticle partitioning in the five lung lobes was dependent on the particle size when the particles were $10 \mathrm{~nm}$ or less in diameter. With larger particles, the partitioning remained steady, regardless of the particle size.

- The upper (right and left) lobar branches attracted a significantly higher percentage $(>70 \%)$ of the inhaled nanoparticles.

- The deposition of larger $(\mathrm{d}>20 \mathrm{~nm})$ nanoparticles was less sensitive to large-scale geometric variations, such as bends and contractions near the tracheal entrance, than to the small-scale superficial roughness of the passage.

- The deposition of nanoparticles was significantly lower in the left and right bronchi (5-8\%) than the trachea (25$45 \%$ ). The deposition efficiency was approximately equal between the models (Fig. 12(c)). The geometric variation near the tracheal entrance produced only negligible effects on the downstream nanoparticle deposition in the first-level bronchi.

\section{ACKNOWLEDGEMENTS}

The financial supports provided by the National Natural Science Foundation of China (Grant No. 91643102) and the Australian Research Council (Grant Nos. DE 180101138 and DP160101953) are gratefully acknowledged.

\section{REFERENCES}

Asgharian, B. and Price, O.T. (2007). Deposition of ultrafine (nano) particles in the human lung. Inhalation Toxicol. 19: 1045-1054. https://doi.org/10.1080/08958370701626501

Balásházy, I., Hofmann, W. and Heistracher, T. (1999). Computation of local enhancement factors for the quantification of particle deposition patterns in airway bifurcations. J. Aerosol Sci. 30: 185-203. https://doi.org/ 10.1016/S0021-8502(98)00040-8

Cheng, Y.S, Zhou, Y. and Chen, B.T. (1999). Particle deposition in a cast of human oral airways. Aerosol Sci. Technol. 31: 286-300. https://doi.org/10.1080/027868299304165

Cohen., B.S., Sussman, R.G. and Lippmann, M. (1990). Ultrafine particle deposition in a human tracheobronchial cast. Aerosol Sci. Technol. 12: 1082-1091. https://doi.org/ $10.1080 / 02786829008959418$
Comer, J.K., Kleinstreuer, C. and Kim, C.S. (2001). Flow structures and particle deposition patterns in doublebifurcation airway models. Part 2: Aerosol transport and deposition. J. Fluid Mech. 435: 55-80. https://doi.org/10. 1017/S0022112001003810

Dong, J., Shang, Y., Tian, L., Inthavong, K., Qiu, D. and Tu, J. (2019a). Ultrafine particle deposition in a realistic human airway at multiple inhalation scenarios. Int. J. Numer. Methods Biomed. Eng. 35: e3215:1-15. https://doi.org/10.1002/cnm.3215

Dong, J., Tian, L. and Ahmadi, G. (2019b). Numerical assessment of respiratory airway exposure risks to diesel exhaust particles. Exp. Comput. Multiphase Flow 1: 5159. https://doi.org/10.1007/s42757-019-0005-2

Frederix, E.M.A., Kuczaj, A.K., Nordlund, M., Bělka, M., Lizal, F., Jedelský, J., Elcner, J., Jícha, M. and Geurts, B.J. (2018). Simulation of size-dependent aerosol deposition in a realistic model of the upper human airways. $J$. Aerosol Sci. 115: 29-45. https://doi.org/10.1016/j.jaeros ci.2017.10.007

Gu, X., Wen, J., Wang, M., Jian, G., Zheng, G. and Wang, S. (2019). Numerical investigation of unsteady particle deposition in a realistic human nasal cavity during inhalation. Exp. Comput. Multiphase Flow 1: 39-50. https://doi.org/10.1007/s42757-019-0007-0

Häußermann, S., Bailey, A.G., Bailey, M.R., Etherington, G. and Youngman, M. (2002). The influence of breathing patterns on particle deposition in a nasal replica cast. $J$. Aerosol Sci. 33: 923-933. https://doi.org/10.1016/S00218502(02)00044-7

Heistracher, T. and Hofmann, W. (1995). Physiological realistic models of bronchial airway bifurcations. $J$. Aerosol Sci. 26: 497-509. https://doi.org/10.1016/00218502(94)00113-D

Inthavong, K., Choi, L.T., Tu, J., Ding, S. and Thien, F. (2010). Micron particle deposition in a tracheobronchial airway model under different breathing conditions. Med. Eng. Phys. 32: 1198-1212. https://doi.org/10.1016/j.med engphy.2010.08.012

Inthavong, K., Shang, Y. and Tu, J. (2014). Surface mapping for visualization of wall stresses during inhalation in a human nasal cavity. Respir. Physiol. Neurobiol. 190: 5461. https://doi.org/10.1016/j.resp.2013.09.004

Kitaoka, H., Takaki, R. and Suki, B. (1999). A threedimensional model of the human airway tree. J. Appl. Physiol. 87: 2207-2217. https://doi.org/10.1152/jappl.19 99.87.6.2207

Kitaoka, H., Koc, S., Tetsumoto, S., Koumo, S., Hirata, H. and Kijima, T. (2013). 4D model generator of the human lung, "Lung4Cer". 35 th Annual International Conference of the IEEE Engineering in Medicine and Biology Society (EMBC). pp. 453-456. https://doi.org/10.1109/E MBC.2013.6609534

Kleinstreuer, C. and Zhang, Z. (2003). Targeted drug aeroso deposition analysis for a four-generation lung airway model with hemispherical tumors. J. Biomech. Eng. 125: 197-206. https://doi.org/10.1115/1.1543548

Koullapis, P.G., Kassinos, S.C., Bivolarova, M.P. and Melikov, A.K. (2016). Particle deposition in a realistic 
geometry of the human conducting airways: Effects of inlet velocity profile, inhalation flow rate and electrostatic charge. J. Biomech. 49: 2201-2212. https://doi.org/10.10 16/j.jbiomech.2015.11.029

Li, A. and Ahmadi, G. (1992). Dispersion and deposition of spherical particles from point sources in a turbulent channel flow. Aerosol Sci. Technol. 16: 209-226. https://doi.org/10.1080/02786829208959550

Lintermann, A. and Schröder, W. (2017). Simulation of aerosol particle deposition in the upper human tracheobronchial tract. Eur. J. Mech. B. Fluids 63: 73-89. https://doi.org/10.1016/j.euromechflu.2017.01.008

Longest, P.W. and Holbrook, L.T. (2012). In silico models of aerosol delivery to the respiratory tract-development and applications. Adv. Drug Delivery Rev. 64: 296-311. https://doi.org/10.1016/j.addr.2011.05.009

Martonen, T.B., Yang, Y. and Xue, Z.Q. (1994). Influences of cartilaginous rings on tracheobronchial fluid dynamics. Inhalation Toxicol. 6: 185-203. https://doi.org/10.3109/0 8958379408995231

Phalen, R.F. and Raabe, O.G. (2016). The evolution of inhaled particle does modeling: A review. J. Aerosol Sci. 99: 7-13. https://doi.org/10.1016/j.jaerosci.2015.12.008

Phillips, C.G. and Kaye, S.R. (1997). On the asymmetry of bifurcations in the bronchial tree. Respir. Physiol. 107: 85-98. https://doi.org/10.1016/S0034-5687(96)02506-6

Shang, Y., Tian, L., Fan, Y., Dong, J., Inthavong, K. and Tu, J. (2018). Effect of morphology on nanoparticle transport and deposition in human upper tracheobronchial airways. J. Comput. Multiphase Flows 10: 83-96. https://doi.org/ $10.1177 / 1757482 X 18756012$

Smith, S., Cheng, Y-S. and Yeh, H. C. (2001). Deposition of ultrafine particles in human tracheobronchial airways of adults and children. Aerosol Sci. Technol. 35: 697709. https://doi.org/10.1080/02786820152546743

Su, W.C. and Cheng, Y.S. (2015). Estimation of Carbon nanotubes deposition in a human respiratory tract replica. J. Aerosol Sci. 79: 72-85. https://doi.org/10.1016/j.jaero sci.2014.09.005

Su, W.C., Ku, B.K., Kulkarni, P. and Cheng, Y.S. (2016). Deposition of graphene nanoparticle in human upper airways. J. Occup. Environ. Hyg. 13: 48-59. https://doi.org/ 10.1080/15459624.2015.1076162

Tian, L. and Ahmadi, G. (2007). Particle deposition in turbulent duct flows - comparisons of different model predications. J. Aerosol Sci. 38: 377-397. https://doi.org/ 10.1016/j.jaerosci.2006.12.003
Tian, L. and Ahmadi, G. (2012). Transport and deposition of micron and nano particles in human tracheobronchial tree by an asymmetric multi-level bifurcation model. $J$. Comput. Multiphase Flows 4: 159-182. https://doi.org/1 0.1260/1757-482X.4.2.159

Tian, L., Shang, Y., Chen, R., Bai, R., Chen, C., Inthavong, K. and Tu, J. (2017a). A combined experimental and numerical study on upper airway dosimetry of inhaled nanoparticles from an electrical discharge machine shop. Part. Fibre Toxicol. 14: 1-18. https://doi.org/10.1186/s1 2989-017-0203-7

Tian, L., Shang, Y., Dong, J., Inthavong, K. and Tu, J. (2017b). Human nasal olfactory deposition of inhaled nanoparticles at low to moderate breathing rate. $J$. Aerosol Sci. 113: 189-200. https://doi.org/10.1016/j.jaer osci.2017.08.006

Tian, L., Shang, Y., Chen, R., Bai, R., Chen, C., Inthavong, K. and Tu, J. (2019). Correlation of regional deposition dosage for inhaled nanoparticles in human and rat olfactory. Part. Fibre Toxicol. 16: 1-17. https://doi.org/1 0.1186/s12989-019-0290-8

Tu, J., Inthavong, K. and Ahmadi, G. (2013). Computational fluid and particle dynamics in the human respiratory system, Springer Netherlands, New York.

Weibel, E.R. (1963). Morphometry of the human lung, Springer-Verlag Berlin Heidelberg, New York.

Xu, X., Shang, Y., Tian, L., Weng, W. and Tu, J. (2019). Inhalation health risk assessment for the human tracheobronchial tree under PM exposure in a bus stop scene. Aerosol Air Qual. Res. 19: 1365-1376. https://doi.org/10.4209/aaqr.2018.09.0343

Zhang, Y. and Finlay, W.H. (2005). Measurement of the effect of cartilaginous rings on particle deposition in a proximal lung bifurcation model. Aerosol Sci. Technol. 39: 394-399. https://doi.org/10.1080/027868290945785

Zhang, Z., Kleinstreuer, C., Donohue, J.F. and Kim, C.S. (2005). Comparison of micro- and nano-size particle depositions in a human upper airway model. J. Aerosol Sci. 36: 211-233. https://doi.org/10.1016/j.jaerosci.2004.08.006

Zhou, Y. and Cheng, Y.S. (2005). Particle deposition in a cast of human tracheobronchial airways. Aerosol Sci. Technol. 39: 492-500. https://doi.org/10.1080/027868291001385

Received for review, January 12, 2020 Revised, April 10, 2020 Accepted, June 16, 2020 